\title{
The Establishment of Bisphenol A Sensing System Utilizing Molecularly Imprinted Polymer Receptor and Electrochemical Determination
}

\author{
Izumi Kubo, Nobuyuki Yokota, Yuko Nakane, and Yusuke Fuchiwaki \\ Department of Bioinformatics, Graduate School of Engineering, Soka University, 1-236, Tangi-cho, Hachioji, \\ Tokyo 192-8577, Japan \\ Correspondence should be addressed to Izumi Kubo, kubo@t.soka.ac.jp
}

Received 2 October 2010; Accepted 30 October 2010

Academic Editor: Rubin Gulaboski

Copyright () 2011 Izumi Kubo et al. This is an open access article distributed under the Creative Commons Attribution License, which permits unrestricted use, distribution, and reproduction in any medium, provided the original work is properly cited.

A sensing system of bisphenol A (BPA) based on the electrochemical detection utilizing molecularly imprinted polymer (MIP) as a receptor of BPA was investigated. MIP for BPA was polymerized thermally from 4-vinylpyridine as a functional monomer and ethylene dimethacrylate (EDMA) as a cross-linker and served to prepare an MIP packed column. BPA in an aqueous solution was adsorbed to an MIP packed column and eluted by acetonitrile/phosphate buffer $(60 / 40, \mathrm{v} / \mathrm{v})$. From aqueous solution, BPA was adsorbed to the column and eluted completely in the eluent. The eluted BPA was electrochemically detected by cyclic voltammetry. Optimum $\mathrm{pH}$ and scan rate were 7.0 and $0.1 \mathrm{~V} / \mathrm{s}$ in phosphate buffer. Electrochemical detection of BPA in acetonitrile/phosphate buffer was performed, and linear relationship between BPA and anodic peak current was observed at the range of 10-100 $\mu \mathrm{M}$. In the eluent, anodic peak current of BPA was observed around $650 \mathrm{mV}$.

\section{Introduction}

Bisphenol A (BPA) is widely used as a material of plastic container, dishes, and milk bottles and distributed almost everywhere on earth. However, it is known to be an endocrine disrupting chemical (EDC) and its toxicity and biological influences have been reported $[1,2]$. Detection and removal of bisphenol A are quite important.

Conventionally, BPA has been determined with use of GC/MS or HPLC [3]. These apparatuses are expensive and need complicated pretreatment and are not applicable to the on-site monitoring. On the other hand, as electrochemical sensor is simple, inexpensive, and applicable to monitoring.

Molecularly imprinted polymers (MIPs) have been utilized as a solid phase extraction in HPLC. Recently MIPs are utilized as a recognition element of chemical sensors. Chemical sensors to detect triazine moiety such as atrazine and simazine have been reported by coupling of MIPmembrane and electrochemical detection [4-9]. To detect simazine, MIP receptor for simazine was prepared with use of simazine as a template and served to selective binding of simazine in a column [10]. The simazine-imprinted polymer packed column was coupled with the electrochemical determination of simazine and a sensing system of simazine was established by Fuchiwaki et al. [11]. On the other hand, MIP for BPA was extensively studied [12-14] and applied to chromatography in extraction of BPA from environmental water. Electrochemical oxidation of BPA has been studied and applied to its removal from aqueous solution [15]. Sensing system for BPA detection has not been reported based on voltammetry of BPA utilizing MIP as a receptor.

In this study we aimed to establish a sensing system of BPA based on its electrochemical detection, utilizing MIP as a receptor of BPA. To fabricate MIP for BPA, 4vilylpyridine was used as a functional monomer to recognize BPA by hydrogen bond, which was cross-linked by ethylene dimethacrylate (EDMA). And MIP packed column was coupled with electrochemical detection system. BPA was determined through cyclic voltammetry. This system looked simple, reasonable, and user friendly. 


\section{Experimental}

2.1. Materials. BPA, which is a standard chemical for environmental analysis, and 4-vinylpyridine were purchased from Kanto Chemicals. Dibutyl phthalate, 2, 2' -azobis(2, 4 dimethylvaleronitril), and Ethylene dimethacrylate (EDMA) were obtained from WAKO Pure Chemicals. Other reagents were laboratory-grade and used without any purification. 4vinylpiridine was distilled to remove stabilizer prior to use.

2.2. Preparation of Molecularly Imprinted Polymer Receptor for $B P A$. Molecularly imprinted polymer for BPA was prepared according to the procedure reported previously in [13] and modified. 4-vinyl pyridine was used as a functional monomer to fabricate the recognition site for BPA by the hydrogen bonding and cross-liked by EDMA. All the reagents were dissolved by toluene and polymerized at $60^{\circ} \mathrm{C}$. The obtained polymer was crushed well into particles. The particles were thoroughly washed to remove template molecule by methanol and dried in vacuo. In the preparation of MIP, $1 \mathrm{~g}$ of MIP was obtained from $90 \mathrm{mg}$ of BPA, $320 \mathrm{mg}$ of 4-vinylpyridine, and $2.5 \mathrm{~mL}$ of EDMA. The dried polymer particles were filtrated and selected, size between 32 and $90 \mu \mathrm{m}$ mesh. These particles were served as MIP receptor for BPA and $10 \mathrm{mg}$ of them were packed in a glass column. Absorbance of BPA to the MIP receptor was evaluated by the UV absorbance at $270 \mathrm{~nm}$ of the loaded and eluted solution of BPA.

2.3. Electrochemical Measurements. A three-electrode cell was used in the electrochemical measurements. A gold electrode (I.D. $=1.6 \mathrm{~mm}$ ) was used as a working electrode. A platinum wire counter and an $\mathrm{Ag} / \mathrm{AgCl}$ reference electrode were used in the electrochemical experiments. Electrochemical measurements were carried out in a glass vial containing $1 \mathrm{~mL}$ of deoxygenated electrolyte solution at room temperature according to the previously reported procedure [15]. Cyclic voltammetry of BPA was measured at a range from $0 \mathrm{~V}$ to $0.8 \mathrm{~V}$. The response to $\mathrm{BPA}$ was obtained from the current difference, with and without BPA at around $0.6 \mathrm{~V}$, which was the anodic peak current of BPA oxidation.

2.4. BPA Sensing System. BPA sensing system was equipped with an MIP packed column to electrochemical measurement system. An aliquot of sample solution containing BPA was loaded to the MIP column. The bound BPA was eluted by $60 \%(\mathrm{v} / \mathrm{v})$ acetonitrile/0.1 M phosphate buffer (pH7.0). The eluent was served to electrochemical measurement.

\section{Results and Discussion}

3.1. Selective Adsorption of BPA to MIP. Aqueous BPA solution $(50 \mu \mathrm{M})$ was loaded to the column and adsorption and elution of BPA were examined by UV spectroscopy. As shown in Figure 1, the loaded BPA was mostly adsorbed to the column, and in the eluent, bound BPA was removed completely. In order to test the selectivity of the column, bisphenol F (BPF) and nonylphenol were examined.

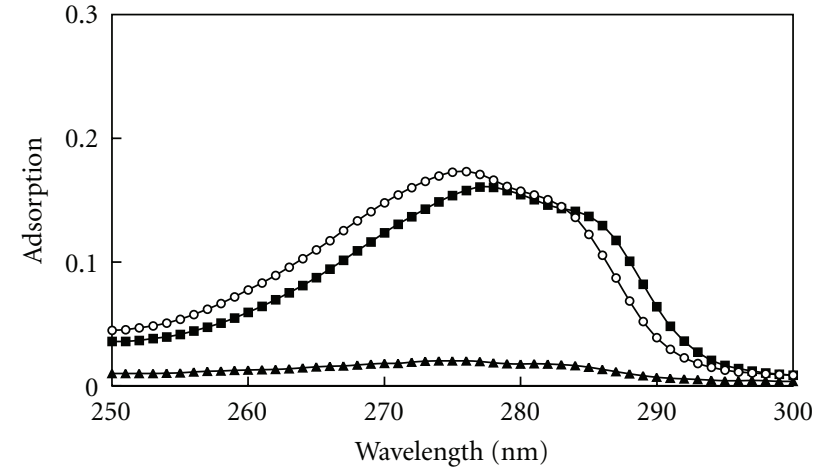

Figure 1: Absorbance of bisphenol A to MIP packed column, and elution from the column $\bigcirc$ loading solution of bisphenol A, $\Delta$ passed through solution, $\mathbf{\square}$ eluent.

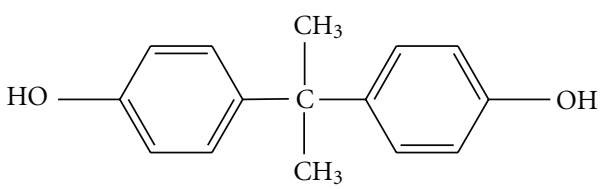

Bisphenol A
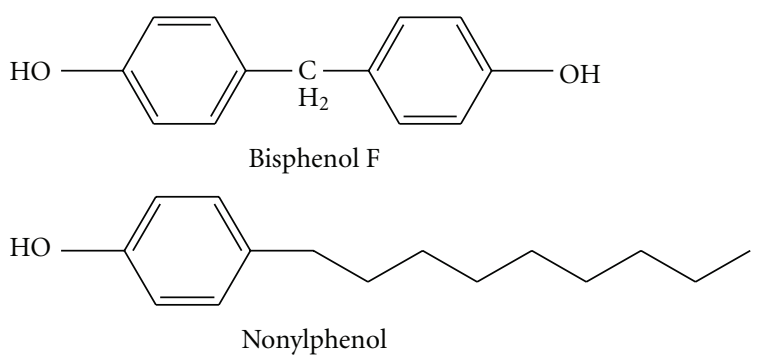

Figure 2: Chemical structures of bisphenol A, bisphenol F, and nonylphenol.

TABLE 1: Selectivity of MIP packed column to bisphenol A.

\begin{tabular}{lccc}
\hline & Bisphenol A & Bisphenol F & Nonylphenol \\
\hline Sample Conc. $(\mu \mathrm{M})$ & 50 & 50 & 50 \\
Adsorption efficiency & 1.0 & 0.80 & 1.0 \\
Elution efficiency & 0.92 & 0.74 & 0.43 \\
\hline
\end{tabular}

The chemical structures of BPA, BPF, and nonylphenol were shown in Figure 2. Fifty $\mu \mathrm{M}$ of these solutions were loaded to the MIP column and checked. Table 1 shows the selectivity of the adsorption and elution. More than $90 \%$ of adsorbed BPA was eluted; however, adsorption of BPF was smaller than BPA and elution of nonylphenol was less than half of BPA. Among them, recovery of BPA from the MIP packed column was the largest. It was favorable for the selective determination of BPA.

3.2. Electrochemical Determination of BPA. Experimental condition of electrochemical measurement of BPA was optimized first. As the condition, $\mathrm{pH}$ of the electrolyte was examined at 4.0, 5.8, and 7.0. At higher $\mathrm{pH}$ than 7.0 electrochemical measurement of BPA was not examined 
because the hydroxyl group of BPA is reported to be partly dissociated to phenolate ion and oxidation of neutral BPA does not take place. Therefore neutral and acidic $\mathrm{pH}$ was examined. Anodic oxidation of BPA was observed as shown in Figure 3, and anodic peak potentials of $\mathrm{pH} 4.0$, 5.8 , and 7.0 were $0.695,0.604$, and $0.520 \mathrm{mV}$, respectively. Peak potentials shifted to lower as to the higher $\mathrm{pH}$. The peak current was the highest at $\mathrm{pH}$ 7.0. Thus in this study, $0.1 \mathrm{M}$ phosphate buffer, $\mathrm{pH} 7.0$, was used. As for the mechanism of electrochemical oxidation of BPA, it was known as the generation of the phenoxy radical and quinone [15].

Next, scan rate was examined (Figure 4). Below the scan rate of $0.1 \mathrm{~V} / \mathrm{sec}$, peak current showed the linear relationship to square root of scan rate. On the other hand, linear relationship was not observed at the scan rate faster than $0.1 \mathrm{~V} / \mathrm{sec}$. These phenomena suggest that at the faster scan rate, electrochemical reaction is not reversible and the side reaction occurs coincidently. In the detection of BPA, cyclic voltammetry was carried out at the scan rate of $0.1 \mathrm{~V} / \mathrm{sec}$ or $0.05 \mathrm{~V} / \mathrm{sec}$ to avoid side reaction.

Under the optimum condition, calibration of BPA was performed. In Figure 5 anodic peak current was plotted against the concentration of BPA in $0.1 \mathrm{M}$ phosphate buffer, $\mathrm{pH} 7.0$, and $60 \%(\mathrm{v} / \mathrm{v})$ acetonitrile in phosphate buffer (eluting solution). Linear relationship $(y=8.8843 x)$ was observed between $10 \mu \mathrm{M}$ to $100 \mu \mathrm{M}$ of BPA in phosphate buffer $\mathrm{pH}$ 7.0. In the eluting solution (60\% of acetonitrile), linear relationship ( $y=6.836 x+0.149)$ was observed at almost the same range of concentration, although the slope was slightly smaller than in phosphate buffer. In eluting solution, at lower concentration than $0.02 \mathrm{mM}$, larger peak current was observed than in phosphate buffer. Electrolyte in acetonitrile worked to help the electrochemical oxidation especially at lower concentration. Then the apparent intercept of the calibration line of BPA in acetonitrile was larger than in phosphate buffer.

3.3. Determination of BPA in the Sensing System. As BPA was electrochemically measured in eluting solution, BPA was determined, using the system coupled with the MIP column. One ml of sample solution containing $50 \mu \mathrm{M}$ of BPA was loaded to the column and the path-thorough solution and eluent was electrochemically measured. As shown in Figure 6 distinguished peak to passed-through solution was not observed; however, in the eluent, anodic peak at around $0.6 \mathrm{~V}$ was clearly observed, and $10 \mu \mathrm{M}$ of BPA was detected too. Observed peak current almost agreed with that observed in $60 \%$ acetonitrile solution. As for the selectivity in anodic response, nonylphenol showed a different response in cyclic voltammetry. BPA would be easily distinguished from other phenols except bisphenols.

\section{Conclusion}

With the sensing system composed of the MIP packed column and an electrochemical analyzer, BPA was easily

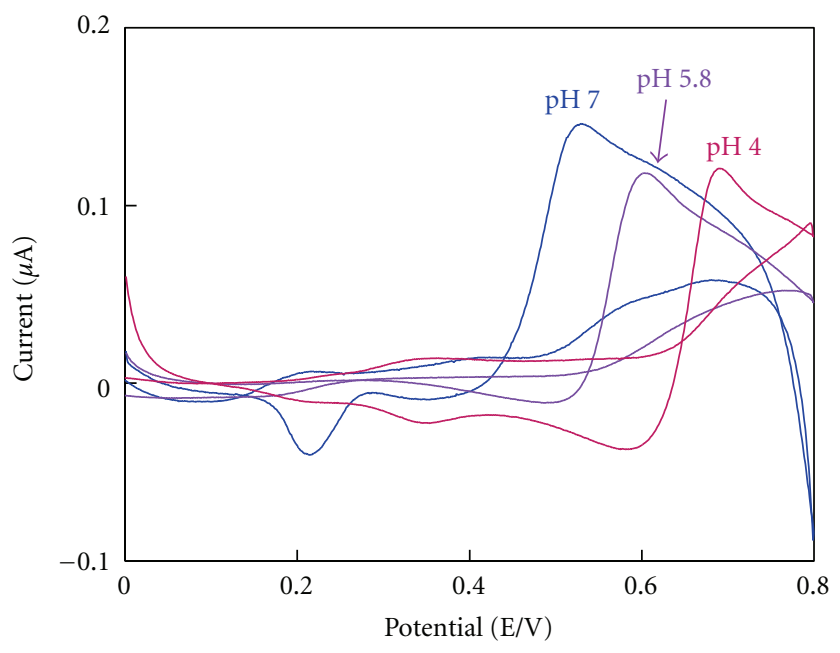

FIGURE 3: Effect of $\mathrm{pH}$ to the anodic oxidation of bisphenol A. Cyclic voltammogram of $0.02 \mathrm{mM}$ bisphenol A. scan rate $0.1 \mathrm{~V} / \mathrm{sec}$.

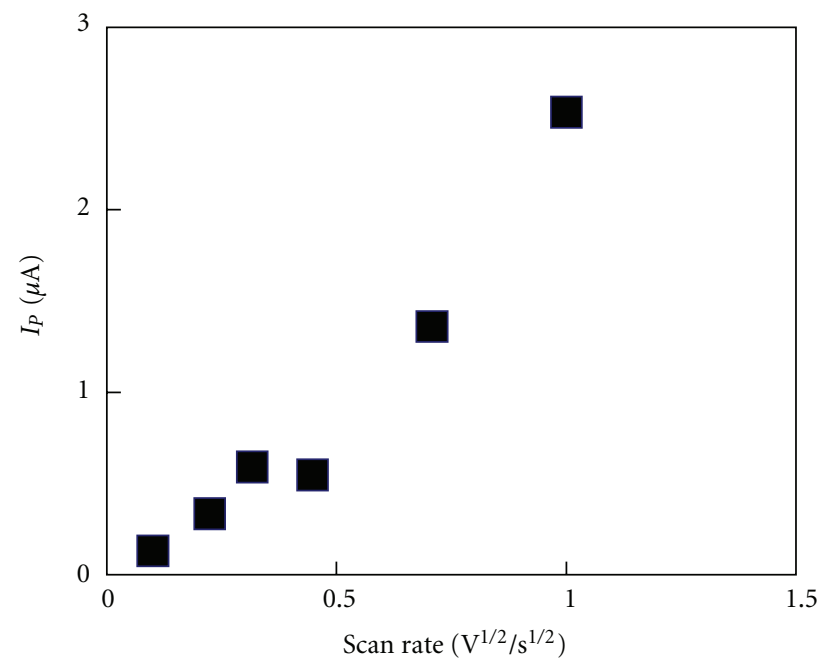

FIGURE 4: Effect of scan rate to the anodic oxidation of bisphenol A. Anodic peak potentials of $0.1 \mathrm{mM}$ bisphenol $\mathrm{A}$ at $\mathrm{pH} 7.0$ were plotted.

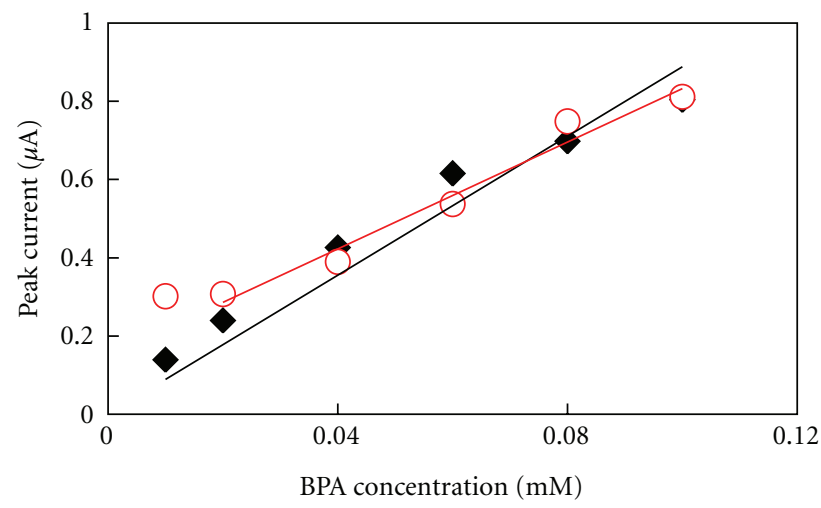

Figure 5: Calibration plot of bisphenol A. $\mathbf{a}$ in $0.1 \mathrm{M}$ phosphate buffer, $\mathrm{pH} 7.0, \bigcirc$ in $60 \%(\mathrm{v} / \mathrm{v})$ acetonitrile/0.1 M phosphate buffer, pH7.0. Scan rate $0.1 \mathrm{~V} / \mathrm{sec}$. 


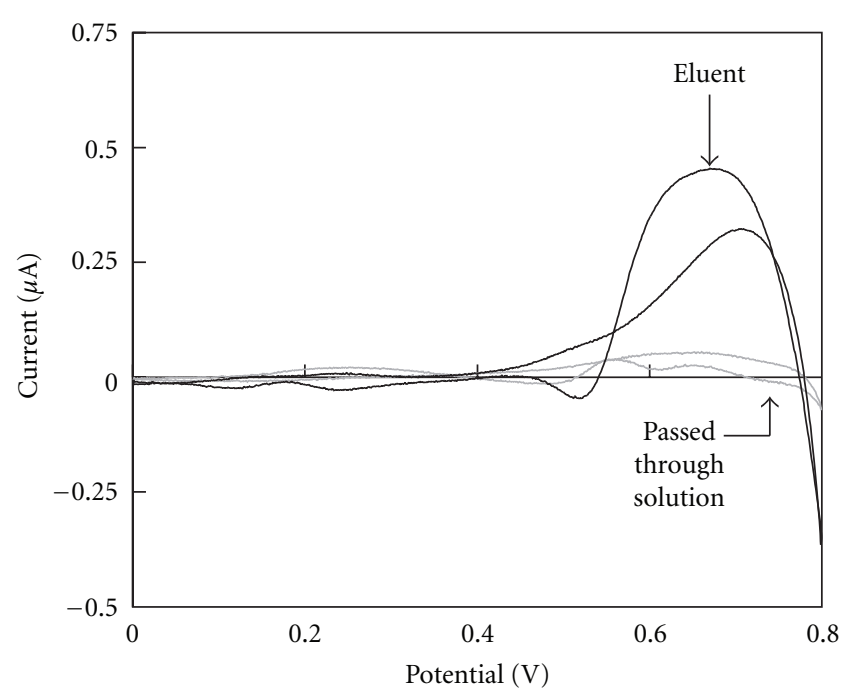

Figure 6: Response of bisphenol A with this sensing system.

determined at the range of $10-100 \mu \mathrm{M}$ of BPA rapidly. This system will be applied to the determination of food or biological fluid in the near future.

\section{Acknowledgments}

This work was supported by a Grant-in-Aids for scientific research from the Ministry of Education, Science, Sports and Culture, Japan. The authors are grateful for helpful support from Dr. Kanamatsu in Soka University.

\section{References}

[1] J. E. Biles, K. D. White, T. P. McNeal, and T. H. Begley, "Determination of the diglycidyl ether of bisphenol A and its derivatives in canned foods," Journal of Agricultural and Food Chemistry, vol. 47, no. 5, pp. 1965-1969, 1999.

[2] R. Pulgar, M. F. Olea-Serrano, A. Novillo-Fertrell et al., "Determination of bisphenol A and related aromatic compounds released from Bis-GMA-based composites and sealants by high performance liquid chromatography," Environmental Health Perspectives, vol. 108, no. 1, pp. 21-27, 2000.

[3] J. Sajiki, K. Takahashi, and J. Yonekubo, "Sensitive method for the determination of bisphenol-A in serum using two systems of high-performance liquid chromatography," Journal of Chromatography B, vol. 736, no. 1-2, pp. 255-261, 1999.

[4] M. T. Muldoon and L. H. Stanker, "Polymer synthesis and characterization of a molecularly imprinted sorbent assay for atrazine," Journal of Agricultural and Food Chemistry, vol. 43, no. 6, pp. 1424-1427, 1995.

[5] J. Matsui, Y. Miyoshi, O. Doblhoff-Dier, and T. Takeuchi, "A molecularly imprinted synthetic polymer receptor selective for atrazine," Analytical Chemistry, vol. 67, no. 23, pp. 4404-4408, 1995.

[6] T. A. Sergeyeva, S. A. Piletsky, A. A. Brovko, E. A. Slinchenko, L. M. Sergeeva, and A. V. El'skaya, "Selective recognition of atrazine by molecularly imprinted polymer membranes. Development of conductometric sensor for herbicides detection," Analytica Chimica Acta, vol. 392, no. 2-3, pp. 105$111,1999$.

[7] C. Luo, M. Liu, Y. Mo, J. Qu, and Y. Feng, "Thickness-shear mode acoustic sensor for atrazine using molecularly imprinted polymer as recognition element," Analytica Chimica Acta, vol. 428, no. 1, pp. 143-148, 2001.

[8] T. Panasyuk-Delaney, V. M. Mirsky, M. Ulbricht, and O. S. Wolfbeis, "Impedometric herbicide chemosensors based on molecularly imprinted polymers," Analytica Chimica Acta, vol. 435, no. 1, pp. 157-162, 2001.

[9] R. Shoji, T. Takeuchi, and I. Kubo, "Atrazine sensor based on molecularly imprinted polymer-modified gold electrode," Analytical Chemistry, vol. 75, no. 18, pp. 4882-4886, 2003.

[10] Y. Fuchiwaki, A. Shimizu, and I. Kubo, "6-Chloro-N,Ndiethyl-1,3,5-triazine-2,4-diamine (CAT) sensor based on biomimetic recognition utilizing a molecularly imprinted artificial receptor," Analytical Sciences, vol. 23, no. 1, pp. 4953, 2007.

[11] Y. Fuchiwaki, N. Sasaki, and I. Kubo, "Development of an electrochemical sensing system for 6-chloro-n,n-diethyl1,3,5-triazine-2,4-diamine (CAT) utilizing an amalgamated gold electrode and artificial sensor receptor," Electrochemistry, vol. 75, no. 9, pp. 709-714, 2007.

[12] V. P. Joshi, M. G. Kulkarni, and R. A. Mashelkar, "Molecularly imprinted adsorbents for positional isomer separation," Journal of Chromatography A, vol. 849, no. 2, pp. 319-330, 1999.

[13] H. Sanbe and J. Haginaka, "Uniformly sized molecularly imprinted polymers for bisphenol A and $\beta$-estradiol: retention and molecular recognition properties in hydro-organic mobile phases," Journal of Pharmaceutical and Biomedical Analysis, vol. 30, no. 6, pp. 1835-1844, 2003.

[14] T. Kubo, K. Hosoya, Y. Watabe et al., "On-column concentration of bisphenol A with one-step removal of humic acids in water," Journal of Chromatography A, vol. 987, no. 1-2, pp. 389-394, 2003.

[15] H. Kuramitz, Y. Nakata, M. Kawasaki, and S. Tanaka, "Electrochemical oxidation of bisphenol A. Application to the removal of bisphenol A using a carbon fiber electrode," Chemosphere, vol. 45 , no. 1 , pp. 37-43, 2001. 


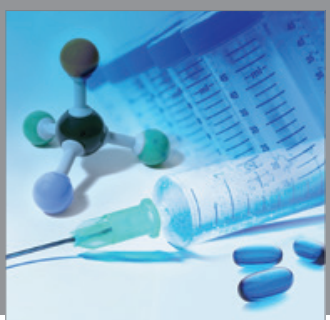

International Journal of

Medicinal Chemistry

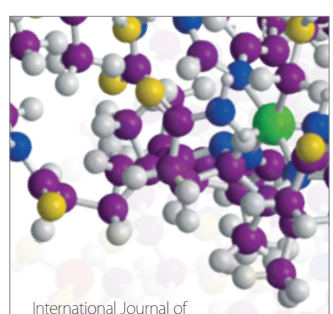

Carbohydrate Chemistry

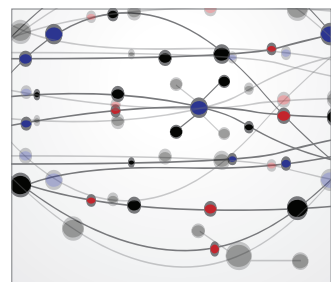

The Scientific World Journal
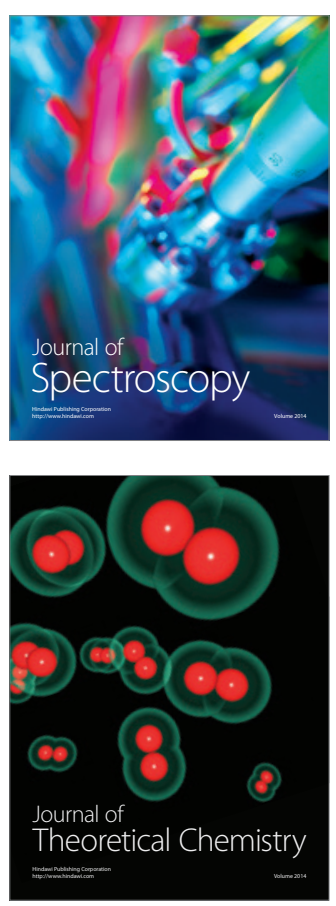
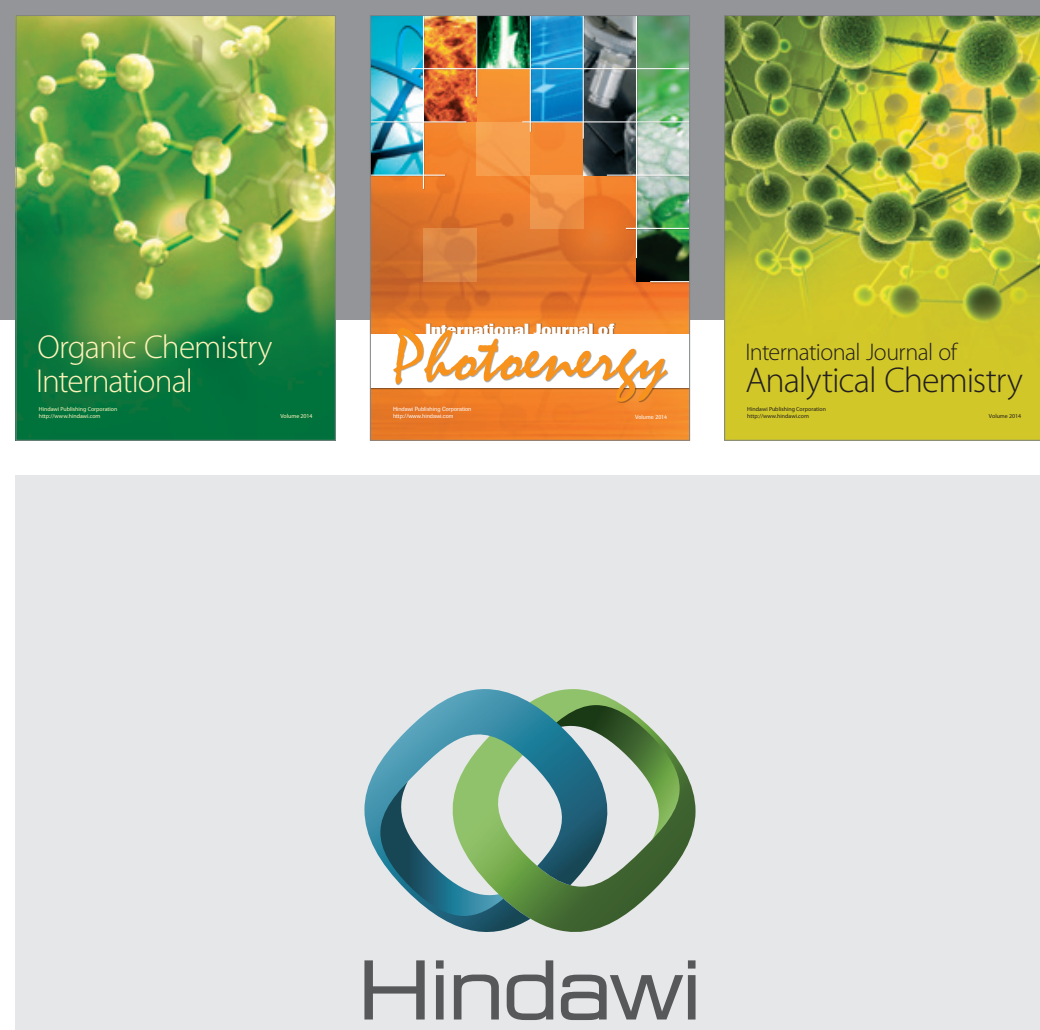

Submit your manuscripts at

http://www.hindawi.com
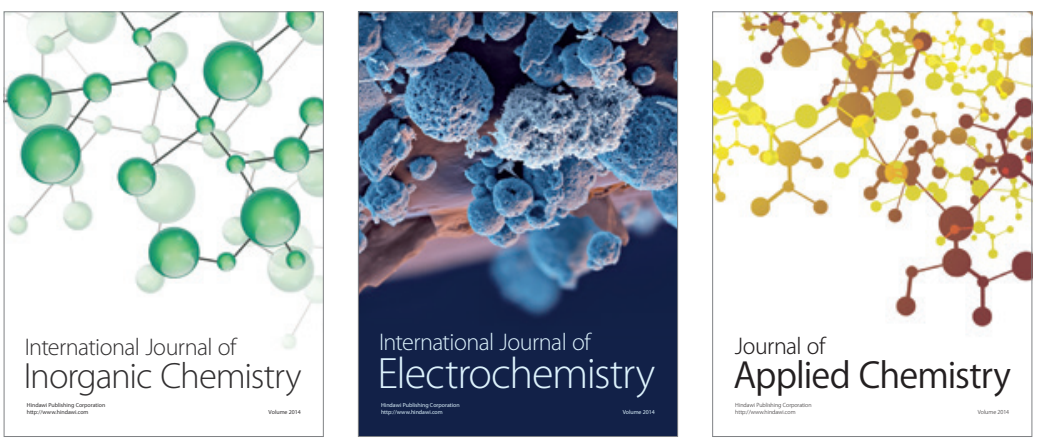

Journal of

Applied Chemistry
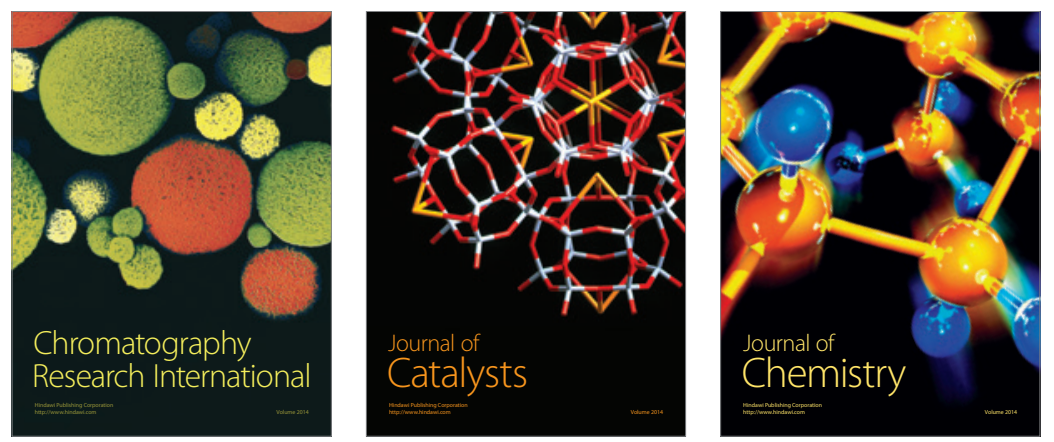
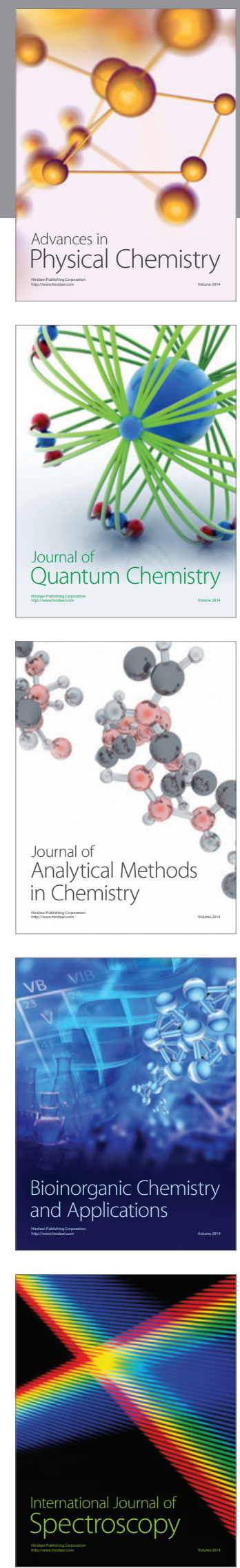